\title{
Evaluación de diferentes reactivos aplicados en la precipitación del oro
}

\section{Evaluation of different reagents applied in gold precipitation}

Javier Ignacio Briones García. ${ }^{1}$, Norma del Rocío Toledo Castillo. ${ }^{2}$, \& Teresita Jackelin Mejía Reinoso. ${ }^{3}$

Recibido: 11-02-2020 / Revisado: 18-03-2020 /Aceptado: 04-04-2020/ Publicado: 08-05-2020

\begin{abstract}
.
DOI: https://doi.org/10.33262/concienciadigital.v3i2.1.1234

In the present research work, 4 different reagents used in the precipitation of gold from solutions are evaluated, two of them used in a classical way such as sodium bisulfite and ferrous sulfate, against two rare species such as sugar and starch. In the experimental part, alluvial gold was mixed with sandy river material to assess the efficiency of the reagents, the leaching of the gold was carried out with aqua regia, this being a particularly strong oxidizing agent formed by the mixture of nitric acid and hydrochloric acid In a 3: 1 volume ratio respectively, statistical programming software was applied in the data analysis, with sugar being the highest precipitating agent.
\end{abstract}

Keywords: Precipitation. Gold. Sodium bisulfite. Ferrous sulfate. Sugar. Starch

\section{Resumen.}

En el presente trabajo de investigación se evalúa 4 diferentes reactivos utilizados en la precipitación del oro desde soluciones, dos de ellos utilizados de forma clásica como son el bisulfito de sodio y el sulfato ferroso, frente a dos especies poco comunes como el azúcar y el almidón. En la parte experimental se mezcló oro de aluvión con material arenoso de río para valorar la eficiencia de los reactivos, la lixiviación del oro se llevó

\footnotetext{
${ }^{1}$ Escuela Superior Politécnica de Chimborazo, Sede Morona Santiago. Macas, Ecuador. javier.briones@espoch.edu.ec

2 Escuela Superior Politécnica de Chimborazo, Sede Morona Santiago. Macas, Ecuador. toledo@espoch.edu.ec

${ }^{3}$ Escuela Superior Politécnica de Chimborazo, Maestría en química aplicada, Estudiante de posgrado. Macas, Ecuador. jmejiareinoso@yahoo.com
} 
a cabo con agua regia siendo este un agente oxidante particularmente fuerte formado por la mezcla de ácido nítrico y ácido clorhídrico en una proporción de volumen 3:1 respectivamente, en el análisis de datos se aplicó un software de programación estadístico, siendo el azúcar el mayor agente precipitante.

Palabras claves: Precipitación. Oro. Bisulfito de sodio. Sulfato ferroso. Azúcar. Almidón.

\section{Introducción.}

El oro ha sido durante mucho tiempo tema de investigación científica, solo alrededor del $0.1 \%$ de todo el oro contenido en la corteza terrestre está disponible, al menos teóricamente, para la minería, pero estas partículas naturales son raras, en su forma natural es monoisotópico y en condiciones normales es inerte a lo orgánico, tiene una red cúbica centrada y no sufre transformaciones alotrópicas, se caracteriza por un bajo valor de resistencia a la tracción $\mathrm{s}$ - del orden de $13-13.3 \mathrm{kp} / \mathrm{mm}$ - y un alto valor de alargamiento - del orden del 50\% - en estado recocido. El límite elástico también es muy bajo, es de 0,35 $\mathrm{kp} / \mathrm{mm}$ (Boshin et al., 2002)

El método más antiguo para extraer metales raros, basado en la alta densidad del oro, es lavar las arenas que las contiene. "La minería ... el lavado de oro requiere solo medios mecánicos, y por lo tanto no es de extrañar que el oro fuera conocido incluso por los salvajes en los tiempos históricos más antiguos" (D.I. Mendeleev.). Pero la existencia de la hidrometalurgia permite extraer el oro en forma de disoluciones, siendo la lixiviación uno de los métodos más utilizados, el cianuro es un ejemplo claro de ello, aunque en los últimos años se han tratado de encontrar otras alternativas.

\section{Interacción de los metales con ácidos}

Casi todos los metales son oxidados por ácidos. En la mayoría de los casos, las películas protectoras de óxido que exhiben una naturaleza básica o anfótera son fácilmente solubles en ácidos. Además, el proceso redox de la interacción de un metal con un ácido está determinado tanto por la actividad del metal como por la naturaleza y concentración del ácido (Tretyakov et al. 1993). Los ácidos clorhídrico y sulfúrico diluidos, oxidan solo aquellos metales que están en una serie de tensiones al hidrógeno, ya que el papel del agente oxidante en estos ácidos es desempeñado por los iones de hidrógeno $\mathrm{H}^{+}$, que se reducen a hidrógeno libre (Pustovalova L.M. y Nikanoroba I.E. 2005)

Los átomos de metal tienen pocos electrones en la capa externa, en la cantidad de 1, 2 o 3. Los metales exhiben propiedades electropositivas y tienen baja electronegatividad, menos de dos. Para la interacción de los ácidos con el metal, se deben cumplir ciertas condiciones (en contraste con las reacciones de los ácidos con bases y óxidos básicos, que ocurren casi siempre). Primero, el metal debe ser suficientemente activo (reactivo) con respecto a los ácidos. Por ejemplo, el oro, la plata, el cobre, el mercurio y algunos otros metales, con la 
liberación de hidrógeno no reaccionan con los ácidos. Los metales como el sodio, el calcio y el zinc, por el contrario, reaccionan muy activamente con la liberación de hidrógeno gaseoso y una gran cantidad de calor. Según la reactividad con respecto a los ácidos, todos los metales están dispuestos en una serie de actividad metálica. A la izquierda están los metales más activos, a la derecha están inactivos. Cuanto más a la izquierda hay un metal en una serie de actividades, más intensamente interactúa con los ácidos. En segundo lugar, el ácido debe ser lo suficientemente fuerte como para reaccionar con el metal. Por la fuerza de un ácido se entiende su capacidad para dar iones de hidrógeno $\mathrm{H}^{+}$. Por ejemplo, los ácidos vegetales (málico, cítrico, oxálico, etc.) son ácidos débiles y reaccionan muy lentamente con metales como zinc, cromo, hierro, níquel, estaño, plomo (aunque pueden reaccionar con bases y óxidos metálicos) (Karapetyants M.Kh. y Drakin S.I. 2000).

\section{Agua regia}

Es un agente oxidante particularmente fuerte formado por la mezcla de ácido nítrico y ácido clorhídrico con concentraciones del 65\% y 35\% respectivamente, en una proporción de una a tres partes por volumen (Kreshkov A.P. y Yaroslavtsev A.A. 1960). La unión de HCl y $\mathrm{HNO}^{3}$ produce una mezcla compleja de productos altamente activos, incluidos los asociados, dióxido de nitrógeno, cloro y cloruro de nitrosilo, esta se prepara inmediatamente antes de su uso: durante el almacenamiento, se descompone fácilmente y es poco estable formando productos gaseosos, la formación de dióxido de nitrógeno y cloruro de nitrosilo da el color del agua regia siendo este un líquido amarillo con olor a cloro y óxidos de nitrógeno.

La efectividad del agua regia como agente oxidante se asocia en gran medida con una disminución en el potencial de oxidación del metal debido a la formación de compuestos complejos de cloro. La formación de este complejo oxidante en un entorno fuertemente ácido permite disolver incluso metales de baja actividad como el oro, el platino y el paladio, incluso a temperatura ambiente (Mihaylenko Y. I. 1970)

El mecanismo de formación del agua regia se puede representar de la siguiente manera.

- El ácido nítrico $\mathrm{HNO}_{3}$ oxida al ácido clorhídrico $\mathrm{HCl}$, siendo un proceso reversible el cual procede de acuerdo con la ecuación (1).

$$
\mathrm{HNO}_{3}+3 \mathrm{HCl} \leftarrow \rightarrow \mathrm{NOCl}+\mathrm{Cl}_{2}+2 \mathrm{H}_{2} \mathrm{O}(1)
$$

- Se habla del agua regia como un agente oxidante muy fuerte debido al cloruro de nitrosilo $(\mathrm{NOCl})$, que se descompone con la liberación de cloro atómico:

$$
2 \mathrm{NOCl} \leftarrow \rightarrow 2 \mathrm{NO}+\mathrm{Cl}_{2}(2)
$$

Debido a esta liberación se disuelve la mayoría de los metales, incluido el oro, por lo que los alquimistas lo llamaron agua regia, ya que el oro era considerado el "rey de los metales". 
No disuelve rodio (Rh), tántalo (Ta), iridio (Ir) y titanio (Ti).

Durante la interacción del oro con el agua regia, el cloruro de nitrosilo se descompone en óxido nítrico y cloro atómico, el cual oxida los átomos y iones de oro.

$$
\mathrm{Au}+\mathrm{NOCl} \leftarrow \rightarrow \mathrm{AuCl}+\mathrm{NO}(3)
$$

Después ocurre una nueva reacción redox, formando cloruro de oro:

$$
\mathrm{AuCl}+\mathrm{Cl}_{2} \leftarrow \rightarrow \mathrm{AuCl}_{3}(4)
$$

El cloruro de oro resultante se une inmediatamente con otra molécula de $\mathrm{HCl}$, formando tetracloroácido, que se lo conoce comúnmente como "oro de cloro":

$$
\mathrm{AuCl}_{3}+\mathrm{HCl} \leftarrow \rightarrow \mathrm{H}\left(\mathrm{AuCl}_{4}\right)(5)
$$

Este complejo ácido se cristaliza con cuatro moléculas de agua en forma de $\mathrm{H}\left(\mathrm{AuCl}_{4}\right) .4 \mathrm{H}_{2} \mathrm{O}$. Sus cristales son de color amarillo claro; la solución acuosa también es de color amarillo. Si se calienta cuidadosamente con ácido clorhídrico, se descompone con la liberación de $\mathrm{HCl}$ y cristales de color marrón rojizo de cloruro de oro (III) $\mathrm{AuCl}_{3}$. Todos los compuestos de oro se descomponen fácilmente cuando se calientan para liberar oro metálico (Dimova M.C. y Malinina R. D. 1989).

La ecuación de reacción total del oro con el agua regia es:

$$
\mathrm{Au}+4 \mathrm{HCl}+\mathrm{HNO}_{3} \leftarrow \rightarrow \mathrm{H}\left[\mathrm{AuCl}_{4}\right]+\mathrm{NO}+2 \mathrm{H}_{2} \mathrm{O}(6)
$$

Incluso Geber, un alquimista árabe de la Edad Media, demostró que el cloro es el principal elemento en el agua regia. El que queda en el aire o durante el almacenamiento a largo plazo se evapora y deja de actuar sobre el oro. La velocidad de disolución del oro en agua regia es de aproximadamente $10 \mu \mathrm{m} / \mathrm{min}$ (Metelskaya G.C. et al. 1980).

\section{Metodología.}

En el presente trabajo de investigación se evaluó la efectividad de 2 reactivos precipitantes utilizados de forma clásica en la recuperación del oro frente a dos especies poco comunes (azúcar y almidón).

Para cada reactivo se generaron 5 repeticiones determinando un promedio general de cementación, en los ensayos se utilizó 30 gramos de material arenoso de río, añadiendo un 
gramo de una muestra previamente pesada de oro de aluvión con una granulometría en el orden de 20 a 200 micras. El análisis de los resultados se logra en el programa Rstudio.

\section{Preparación de la muestra.}

La limpieza de la muestra se llevó a cabo mediante ebullición en ácido nítrico, filtrando posteriormente el material, después de la limpieza, se agrega ácido clorhídrico $\mathrm{HCl}$ en una proporción de volumen 3:1 con diferencia al ácido nítrico. En este caso, el oro se disuelve solo en ácido clorhídrico. Ni el nitrógeno ni el oxígeno están incluidos en el ácido clorhídrico, luego se calienta la mezcla y agrega gradualmente el ácido nítrico y una vez finalizada la disolución, debe mantenerse la disolución calentada durante aproximadamente media hora. El ácido nítrico actúa solo como un agente oxidante, catalizando la entrada de oro en la reacción.

Lo principal es no exagerar con ácido nítrico, ya que, al precipitar el oro de una solución, será necesario deshacerse de él de manera más consistente.

\section{La lixiviación del oro}

Esta se logra con el agua regia, se filtra la solución a través de papel de filtro dejando reposar durante 20-30 minutos. La mayoría de los componentes volátiles se evaporarán y la evaporación llevará menos tiempo, luego, se debe agregar ácido sulfúrico en un volumen del $5 \%$ al de la muestra y se procede con la evaporación de agua regia.

La solución debe evaporarse cuidadosamente, calentando lentamente, pero sin hervir. Cuando se vuelve almibarado, se agrega más ácido clorhídrico y se continúa evaporando, luego, nuevamente agregamos $\mathrm{HCl}$ a la consistencia inicial, y la mezcla resultante se diluye con agua en una proporción de 1:1. Dejamos la solución durante 24 horas en un lugar fresco. Los residuos se depositarán en el fondo. Se filtra cuidadosamente la solución para eliminar los sedimentos y la turbidez.

\section{Precipitación por medio del bisulfito de sodio}

La solución que contenía oro se alcalinizó con urea hasta un ph mayor a 10, luego se agregó 0.79 gramos de bisulfito de sodio por cada gramo de oro disuelto y se calentó en baño maría por una hora, los residuos son lavados con agua destilada, para ser filtrado con papel filtro para ser calcinados.

\section{Precipitación por medio de sulfato ferroso}

Sobre la solución de $\mathrm{AuCl}_{3}$ obtenida de la forma ya descrita anteriormente y contenida en un vaso precipitado, se le agrega la disolución de caparrosa preparada con $5 \mathrm{gr}$ de sulfato ferroso disuelto en 40ml de agua destilada y acidificada al 10\% de ácido sulfúrico, se deja reposar por 30 min y se filtra, el filtro se lava con ácido clorhídrico, agua destilada, agua amoniacal 
y nuevamente con agua destilada, se seca el filtro en una estufa a $70{ }^{\circ} \mathrm{C}$, se calcina y se pesa la muestra.

\section{Precipitación por medio del Azúcar}

Utilizando la solución donde se encuentra disuelto el oro, es necesario alcalinizar a un pH de 11-12, esto se logra con carbonato de calcio carbonizado, para esto se calcina el bicarbonato de sodio y se prepara una alta concentración con agua destilada, se filtra y se agrega 7 gramos de sacarosa por cada gramo de oro se lleva a ebullir y después de un tiempo el oro precipita y se calcina.

\section{Precipitación por medio del Almidón}

Para realizar la precipitación con almidón se prepara una solución al $1 \%$ en una solución de $\mathrm{NaCl}$ al $0.3 \%$, y por cada $5 \mathrm{ml}$ de dicha solución se agrega $1 \mathrm{ml}$ de saliva, para que la enzima amilasa salival pueda transformarla en dextrina a una temperatura de $38^{\circ} \mathrm{C}$, por un tiempo alrededor de 20 min o hasta que la prueba del yodo salga negativa.

Para precipitar el oro se agrega $1 \mathrm{ml}$ a nuestra solución del agua regia y se lleva a ebullición se deja reposar por 48 horas se filtra y se calcina.

\section{Resultados.}

El oro obtenido luego de su precipitación para todas las repeticiones con cada reactivo empleado, se le aplicó el proceso de calcinado obteniendo así el peso total de oro para cada muestra, los cuales se representan en la tabla 1.

Al determinar el promedio general de oro precipitado por los reactivos permitió observar el rendimiento y efectividad de estos, siendo el azúcar el reactivo más efectivo al precipitar la mayor cantidad de oro.

Tabla 1 - Resultado de oro precipitado de los ensayos con los diferentes reactivos

\begin{tabular}{cccccc}
\hline Muestra & $\begin{array}{l}\text { Oro } \\
\text { agregado } \\
(\mathrm{gr})\end{array}$ & $\begin{array}{l}\text { Oro } \\
\text { precipitado } \\
\text { con Bisulfito } \\
\text { de Sodio }(\mathrm{gr})\end{array}$ & $\begin{array}{l}\text { Oro } \\
\text { precipitado } \\
\text { con Sulfato } \\
\text { Ferroso }(\mathrm{gr})\end{array}$ & $\begin{array}{l}\text { Oro } \\
\text { precipitado } \\
\text { con Azúcar } \\
(\mathrm{gr})\end{array}$ & $\begin{array}{l}\text { Oro } \\
\text { precipitado } \\
\text { con Almidón } \\
(\mathrm{gr})\end{array}$ \\
\hline 1 & 1 & 0.958 & 0.958 & 0.956 & 0.702 \\
2 & 1 & 0.943 & 0.964 & 0.984 & 0.683 \\
3 & 1 & 0.932 & 0.976 & 0.967 & 0.703 \\
4 & 1 & 0.956 & 0.956 & 0.964 & 0.674 \\
5 & 1 & 0.963 & 0.974 & 0.985 & 0.686 \\
& Promedio & 0.9504 & 0.9656 & 0.9712 & 0.6896 \\
\hline
\end{tabular}

Fuente: Elaborado por los autores 
Los datos que arroja la tabla 1 fueron introducidos en un software de programación estadístico llamado Rstudio, realizando en este un análisis de varianza.

Para analizar las relaciones entre las repeticiones realizadas para cada reactivo se realizó un diagrama de dispersión el cual se muestra en el gráfico 1, este nos indica que cada reactivo posee una correlación moderada entre sus repeticiones, así como que los reactivos bisulfito de sodio, sulfato ferroso y azúcar presentan rendimiento elevado con promedios semejantes en precipitación frente al almidón.

Figura 1. Oro recuperado con diferentes reactivos

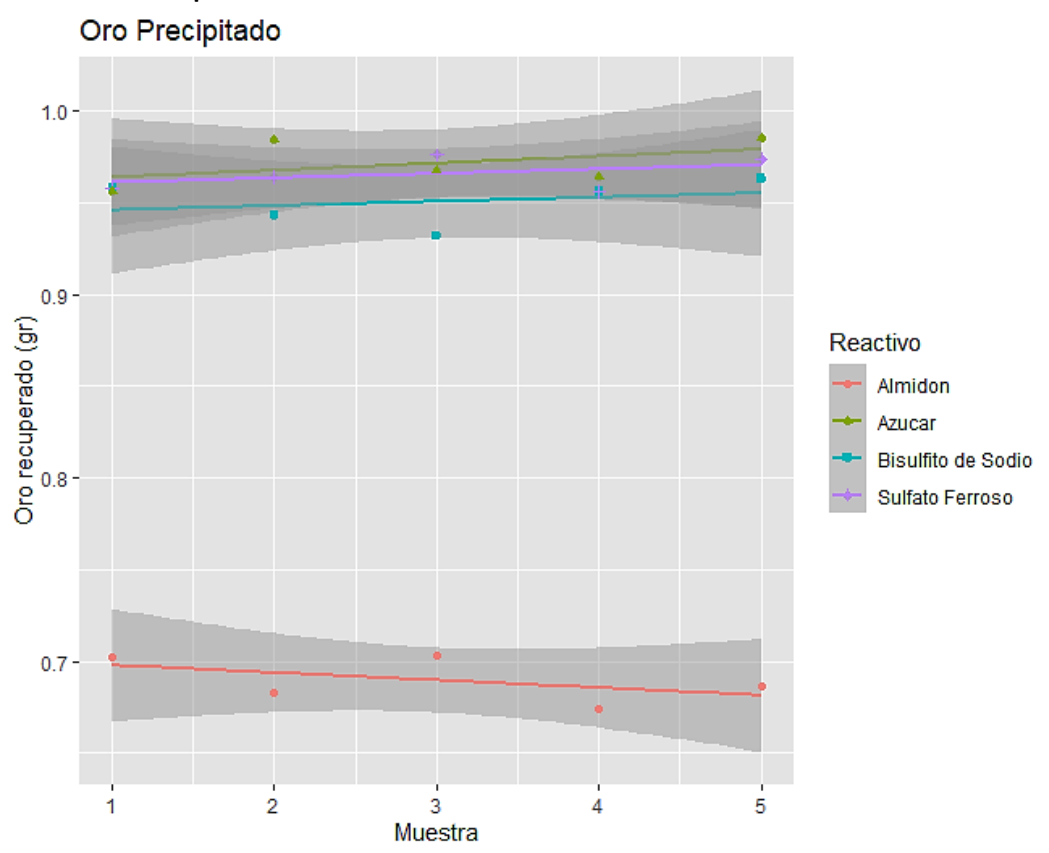

Fuente: Elaborado por los autores

En el programa Rstudio se ingresaron los comandos respectivos para la realización del análisis de la varianza ANOVA con la variable independiente "Oro recuperado" frente a la variable categórica "Reactivo" dando los siguientes resultados:

Terms :

Sum of Squares 0.28023280 .0022604

Deg. of Freedom $\quad 36$

Residua1 standard error: 0.01188592

El programa muestra la suma de los cuadrados y los grados de libertad para los datos ingresados, para determinar el nivel de significancia se ingresó un nuevo comando dando los siguientes resultados:

Df Sum Sq Mean Sq $\mathrm{F}$ value $\operatorname{Pr}(>\mathrm{F})$ 
Reactivo $\quad 3 \quad 0.28023 \quad 0.09341 \quad 661.2<2 \mathrm{e}-16 * * *$

Residuals 160.002260 .00014

$---$

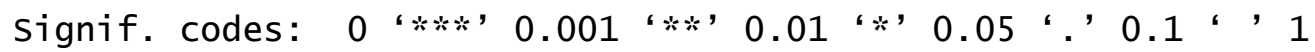

Los datos indican que la prueba si es significativa y que las medias de las repeticiones para cada reactivo son diferentes, para determinar estadísticamente la diferencia de la cantidad de oro recuperado se aplica la prueba de Tukey.

Tukey multiple comparisons of means

95\% family-wise confidence 1 evel

Fit: $\operatorname{aov}($ formula = 'oro recuperado (gr)` $\sim$ Reactivo)

\$Reactivo

Azucar-A1midon

Bisulfito de Sodio-Almidon

Sulfato Ferroso-A7midon

Bisulfito de Sodio-Azucar

$\begin{array}{llllll}\text { Sulfato Ferroso-Azucar } & -0.0056 & -0.027107183 & 0.0159071825 & 0.8774619\end{array}$

$7 w r$

0.2816

0.2608

0.2760

0.260092817

0.239292817

$\begin{array}{llll} & 0.239292817 & 0.2823071825 & 0.0000000\end{array}$

$\begin{array}{lllll}-0.0208 & -0.042307183 & 0.0007071825 & 0.0598138\end{array}$

Los resultados muestran las diferencias de las medias para cada reactivo con su intervalo respectivo y los valores para cada contraste, al comparar las medias del almidón frente a las medias de los demás reactivos se observa que existe una gran diferencia en el porcentaje de oro recuperado, mientras que los demás reactivos presentan similares porcentajes de recuperación, en la figura 2 se muestra un diagrama de cajas donde se observa un resumen de lo descrito.

Figura 2. Comparación de oro recuperado con diferentes reactivos.

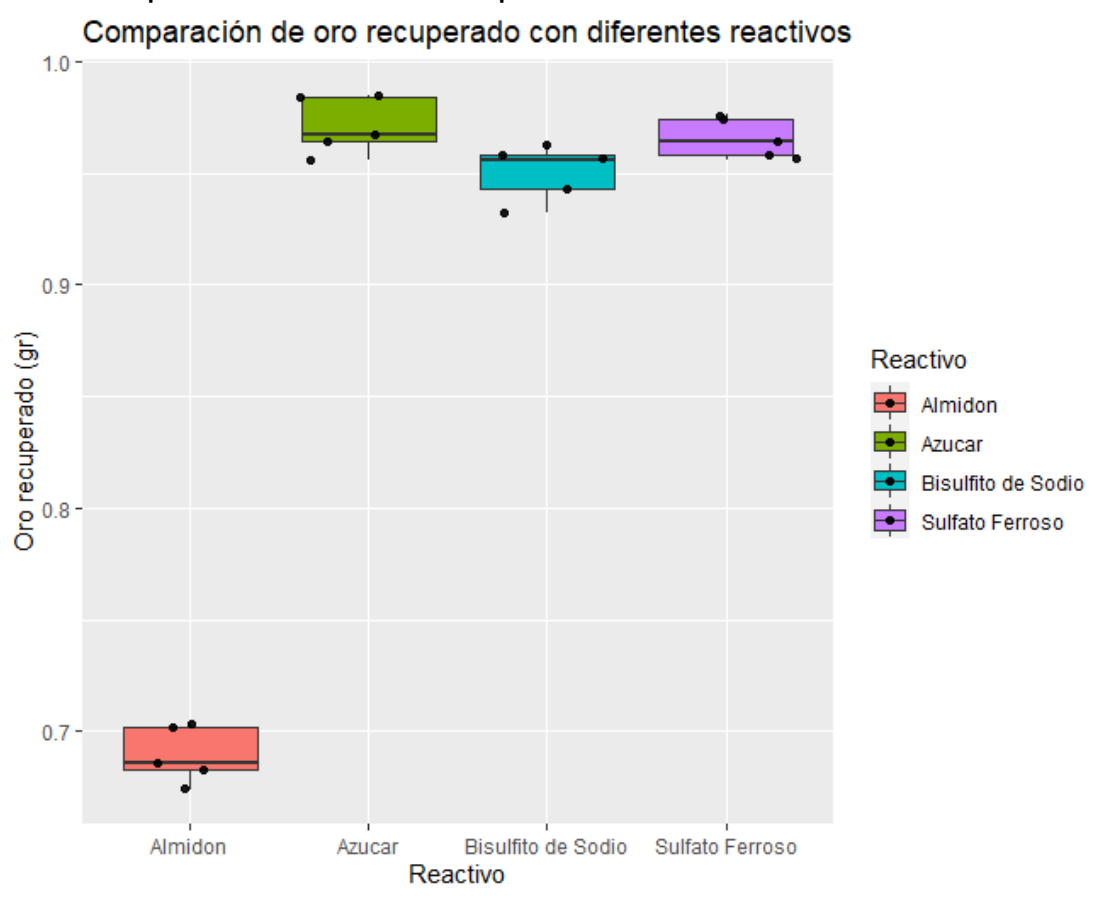

Fuente: Elaborado por los autores 


\section{Conclusiones.}

- La hidrometalúrgica es un proceso de extracción de metales mediante reacciones químicas en soluciones acuosas. Como materias primas, se pueden usar minerales, concentrados químicos, desechos de otras industrias o procesos hidrometalúrgicos. Este método es útil cuando la materia prima contenga una baja concentración de metal y no pueda procesarse por métodos tradicionales. Las características positivas de la hidrometalurgia incluyen la posibilidad de separación de metales de propiedades similares y una opción de procesamiento más simplificada.

- En la actualidad existen diferentes reactivos usados de forma clásica en la recuperación de oro de soluciones, pero nuevos trabajos de investigación presentan una amplia variedad de nuevos agentes precipitantes mas accesibles como es el caso del azúcar y el almidón.

- En las pruebas realizadas se observa que el azúcar presenta un gran rendimiento en la precipitación del oro, pero para esto la solución debe de tener un $\mathrm{pH}$ de 11-12.

- El almidón puede llevar a ser agente precipitante del oro disuelto si se sintetiza adecuadamente en forma de dextrina con enzimas específicas.

\section{Referencias bibliográficas.}

Andrews E. (2012). Diseño del proceso para recuperar Oro y Plata desde depósitos de Relaves de Minera MERIDIAN (tesis de pregrado). Chile.

ÁVILA, V. (2010). Prueba del Oro. Universidad Galileo. FISICC-IDEA. Agosto. CURSO: Química Analítica Cuantitativa (Cuba). Determinación Analítica del Oro y Plata. Facultad deIngeniería Metalúrgica y de Materiales. Cuba.

Bailey, P. R. (1987). Application of activated carbon to gold recovery. The Extractive Metallurgy of South Africa. Ed. Stanley G. G. S. Afr. Inst. Of Min. \& Metall. Monograph series M7, pp. 379 - 611.

Ballester, A., Verdeja, L. y Sancho, J. (2000). Metalurgia Extractiva Volumen I Fundamentos. Madrid, España: Síntesis. pp.333-381,427-468.

Boshin, S., et al. (2002). tecnología de aleación de metales nobles. Moscú, Rusia: Shkola.

Cáceres, A. (1996). Introducción a la hidrometalurgia. Universidad de Atacama. Copiapó, Chile. pp. 3-25,37-43,59-60,77.

Carhuamanca, W. y Cristóbal, B. (2013). Recuperación del Oro mediante la Pre-Aireación en Concentrados de Oro Pirítico en la Empresa Analytica Mineral Services S.A.C. Facultad de Ingeniería Química. Universidad Nacional del Centro del Perú.

Dimova, M.C. y Malinina, R. D. (1989). Metalurgia. Moscú, Rusia: Fenix. 
Dorey, R., Van, D. y Kiel, J. (1988). Overview of heap leaching Technology.In introduction to Evaluation, Design and Operation of Precious Metal Heap Leaching Projects, Littleton, Colorado: Society of Mining Engineers.

Espinoza, L. (2017). Estudio De Investigación Para La Recuperación De Oro Y Plata De Los Relaves De La Planta Veta Dorada S.A.C. Facultad de Ingeniería de Procesos. Universidad Nacional de San Agustín. Arequipa.

Karapetyants, M.H. y Drakin, S.I. (2000). Química general e inorgánica. (4ª ed). Moscú, Rusia: Química.

Mas, M., Aguirre, F. y Amaya, G. (2005). Evaluación de los Procesos de Lixiviación por Agitación Convencional y Carbón en Pulpa en la Hidrometalurgia del Oro. La Serena. Facultad de Ciencias Físicas, Matemáticas y Naturales. San Luis. Argentina.

Marsden, J. y House, I. (1993). The chemistry of gold extraction. Londres, Great Britain: Ellis Horwood Ed.

Martínez M. y José Antonio. (2015). Informe Técnico: Manejo de pilas de Lixiviación de Oro en minera YANACOCHA S.R.L. Lima.

Metelskaya, G., et al. (1980). Química inorgánica. Moscú, Rusia: Química.

Mihaylenko, Y. I. (1970). Curso de química general e inorgánica. Moscú, Rusia: Shkola.

OLIVEROS, H. (2011). Metodología para recuperar metales preciosos: oro, plata y grupo del platino, presentes en desechos electrónicos. Trabajo de Postgrado. Magister en Ingeniería de Materiales y Procesos. Universidad Nacional de Colombia. Facultad de Minas. Medellín. 108 p.

Pustovalova, L. M. y Nikanorova, I. E. (2005). Química inorgánica. Moscú, Rusia: Fenix.

Tretyakov, Yu.D. y Oleinikov, N.N. (1993). Química del procesamiento de materiales. Moscú, Rusia: Educación.

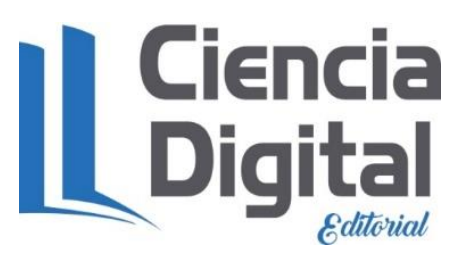


PARA CITAR EL ARTÍCULO INDEXADO.

Briones García, J. I., Toledo Castillo, N. del R., \& Mejía Reinoso, T. J. (2020). Evaluación de diferentes reactivos aplicados en la precipitación del oro . ConcienciaDigital, 3(2.1), 199209. https://doi.org/10.33262/concienciadigital.v3i2.1.1234

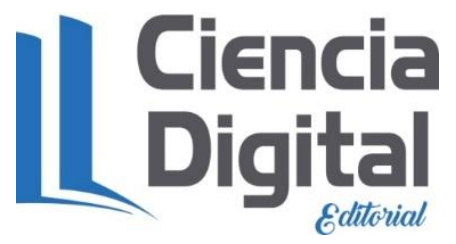

El artículo que se publica es de exclusiva responsabilidad de los autores y no necesariamente reflejan el pensamiento de la Revista Conciencia Digital.

El artículo queda en propiedad de la revista y, por tanto, su publicación parcial y/o total en otro medio tiene que ser autorizado por el director de la Revista Conciencia Digital.
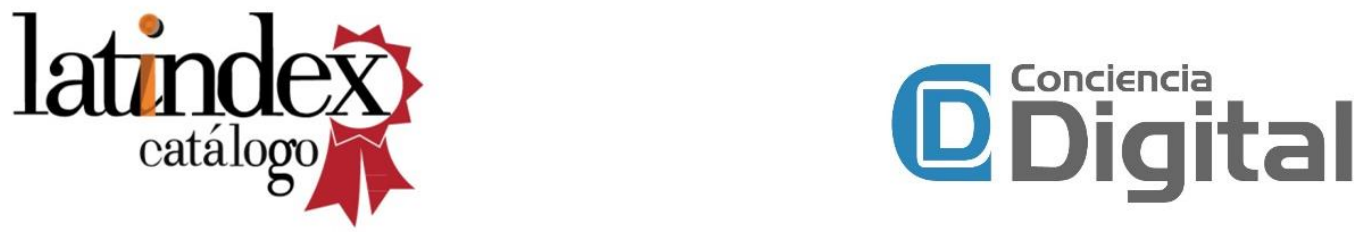